\title{
ORIGINAL ARTICLE Hematological complications in anorexia nervosa
}

\author{
E De Filippo ${ }^{1,3}$, M Marra $^{1}$, F Alfinito ${ }^{1,3}$, ML Di Guglielmo' ${ }^{1}$ P Majorano ${ }^{1}$, G Cerciello ${ }^{1}$, C De Caprio ${ }^{1}$, F Contaldo ${ }^{2}$ and F Pasanisi $^{2}$
}

BACKGROUND/OBJECTIVES: Anemia, leukopenia and, although less frequently, thrombocytopenia are possible hematological complications of anorexia nervosa considered strictly secondary to chronic malnutrition. This is a retrospective study on the prevalence of these disorders in a large cohort of 318 female patients with AN (20.4 \pm 5.6 years, body mass index (BMI) $\left.15.9 \pm 1.6 \mathrm{~kg} / \mathrm{m}^{2}\right), \mathrm{recruited}$ in the Outpatient Unit for Malnutrition secondary to Eating Disorders at the Department of Clinical Medicine and Surgery, Federico II University Hospital, since February 1991 to December 2012.

SUBJECTS/METHODS: Patients were studied on an outpatient basis after obtaining medical history, clinical examination, routine hematobiochemical and endocrine tests, electrocardiography, psychiatric interview and bioelectrical impedance analysis and, in particular, phase angle determination. All patients with other comorbidities, in particular with mean corpuscular volume $<80 \mathrm{fl}$, were excluded for suspected genetic alteration in the synthesis of hemoglobin.

RESULTS: Hematologic data showed that $16.7 \%$ of patients had anemia, $7.9 \%$ neutropenia and $8.9 \%$ thrombocytopenia. These abnormalities were strictly related to the duration of illness $(P=0.028)$, and to protein energy malnutrition, in particular, BMI and phase angle $(P<0.001)$.

CONCLUSIONS: Our study offers description of the incidence of hematologic defects in a selected and large sample of AN female patients, suggesting that its incidence is related to the degree and duration of protein energy malnutrition.

European Journal of Clinical Nutrition (2016) 70, 1305-1308; doi:10.1038/ejcn.2016.115; published online 20 July 2016

\section{INTRODUCTION}

Anemia, leukopenia and, although less frequently, thrombocytopenia are possible hematological complications of anorexia nervosa (AN); ${ }^{1}$ their prevalence has been reported ranging from 21 to $39 \%$ for anemia, 29 to $39 \%$ for leukopenia and 5 to $11 \%$ for thrombocytopenia. ${ }^{1}$ Hematologic disorders related to malnutrition are probably underestimated because of hemoconcentration, a common finding in patients with AN. Usually anemia in AN is normocytic and normochromic and leukopenia is characterized by lymphocytopenia or neutropenia; thrombocytopenia may also be present. ${ }^{1}$ Bone marrow examination reveals, in most severe cases, signs of atrophy and/or hypocellularity; ${ }_{i}^{2}$ gelatinous bone marrow transformation may also be present with increased bone marrow fat. ${ }^{3}$ Hematological alterations in AN are referred as closely related to chronic malnutrition considering that their resolution is usually obtained with nutritional rehabilitation after a variable length of time. ${ }^{1}$ These alterations do not appear totally dependent on micronutrient deficiencies such as vitamins and iron. In fact, blood cytopenia in anorexic patients can also be found in the presence of normal serum concentration of these elements. ${ }^{1}$ On the other hand, normal plasma vitamin and macronutrient concentrations should not be misinterpreted in patients with AN as normal micronutrient and vitamin status, as these values too can be affected by dehydration. ${ }^{4}$ The accuracy of bioelectrical impedance analysis (BIA) is limited in patients with a body mass index (BMI) below $16 \mathrm{~kg} / \mathrm{m}^{2}$ and in patients with $A N{ }^{5,6}$ but phase angle (PA), a parameter of BIA, can be considered an index of the integrity of the cell membrane. PA identifies extra/intracellular water distribution: a low PA being a common finding in severe malnutrition. ${ }^{7}$ Several researches have proved the close relation between PA and nutritional status. PA exhibits a negative correlation with the subjective global assessment score ${ }^{8,9}$ that assesses nutritional status. PA indeed correlates with both the functional index (hand grip strength cirrhosis patients) and nutritional status (albumin, transferrin)..$^{8-10}$ Moreover, PA, hand grip strength and nutritional status are strictly related to survival in advanced cancer patients with severe weight loss. ${ }^{11-13}$

The aim of this study was to evaluate, retrospectively, the incidence of hematologic disorders in a large cohort of patients with $\mathrm{AN}$, the relationship between the degree and duration of protein energy malnutrition, the PA and hematobiochemical parameters.

\section{MATERIALS AND METHODS}

In this cross-sectional study, 318 (mean age $20.4 \pm 5.6$ (age range 14-47) years, (BMI) $\left.15.9 \pm 1.6(10.5-18.4) \mathrm{kg} / \mathrm{m}^{2}\right)$ amenorrhoic female patients affected by AN, consecutively recruited in the Outpatient Unit at the Department of Clinical Medicine and Surgery, Federico II University Hospital, since February 1991 to December 2012, were considered for the study. The study was carried out according to the Declaration of Helsinki, and the protocol was approved by the local ethics committee at Federico II University Hospital. All patients gave their informed consent for participating in the study.

All patients were postpubertal adolescents. Diagnosis of AN was performed by an expert psychiatrist, according to the Diagnostic and Statistical Manual of Mental Disorders, 4th Edition, Text Revision criteria, ${ }^{13}$ and recently confirmed according to the Diagnostic and Statistical Manual of Mental Disorders, 5th Edition. ${ }^{14}$ Exclusion criteria were other comorbidities (with particular attention to immunological diseases) or specific therapies such as iron supplementation. Patients with low mean cellular volume (MCV) and/or low serum ferritin levels were not included to avoid heterozygosity for thalassemia minor, which is quite common in the Mediterranean area. All patients were studied at entry on an outpatient basis after careful review of the patient's medical history, clinical examination, routine biochemical and

\footnotetext{
${ }^{1}$ Department of Clinical Medicine and Surgery 'Federico II' University, Naples, Italy and 'Interuniversity Center for Obesity and Eating Disorders, Medical School, 'Federico II' University, Naples, Italy. Correspondence: Dr M Marra, Department of Clinical Medicine and Surgery, 'Federico II' University, Via S Pansini no. 5, Naples 80131, Italy.

E-mail: marra@unina.it

${ }^{3}$ These authors contributed equally to this work.

Received 8 May 2015; revised 1 April 2016; accepted 3 May 2016; published online 20 July 2016
} 
endocrine tests, electrocardiography (Oimed, 300A, Shangai, China), psychiatric interview and BIA measurements (Human IM Scan DS-Medica, Milan, Italy). They were further subdivided into three subgroups according to the degree of protein energy malnutrition: mild $\left(16.5<\mathrm{BMI}<18.5 \mathrm{~kg} / \mathrm{m}^{2}\right)$, severe $\left(14.5<\mathrm{BMI}<16.4 \mathrm{~kg} / \mathrm{m}^{2}\right)$ and very severe $\left(\mathrm{BMl}<14.4 \mathrm{~kg} / \mathrm{m}^{2}\right)$.

BIA was performed at $50 \mathrm{kHz}$ at a room temperature of $22-25^{\circ} \mathrm{C}$. BIA variables resistance $(R)$, reactance $(\mathrm{Xc})$ and PA were measured in the postabsorptive state, on the non-dominant side of the body, after voiding and after being in the supine position for $20 \mathrm{~min}$.

Samples for hemoglobin determination were collected in EDTA tubes, whereas samples for serum iron, transferrin and ferritin dosages were collected in serum separator tubes. All samples were evaluated with routine laboratory methods. Laboratory technicians were not aware of the clinical conditions of the patients. For hemoglobin analysis, a hematologic analyzer (ADVIA 2120; Siemens, Munich, Germany) was used; serum iron and ferritin levels were determined by spectrophotometry (Modular; Hitachi, Tokyo, Japan); in particular, for ferritin dosage, an immunoenzymatic assay was used. Finally, for transferrin and total iron-binding capacity, a nephelometric method was used.

Statistical analysis consisted in calculating the prevalence of disorders (using standard cut-points) and in evaluating differences between subgroups. Statistical significance of differences was confirmed by $x^{2}$ tests (for differences in prevalence) and by one-way analysis of variance tests (for differences in mean values).

\section{RESULTS}

Table 1 shows anthropometric data and the length of amenorrhea of the female patients under study.

Hematologic data (Table 2) show that $16.7 \%$ of patients with AN were anemic, $7.9 \%$ neutropenic and $8.9 \%$ thrombocytopenic. The combination of two types of cytopenia was present in $8.7 \%$ of patients (anemia and neutropenia 3.8\%, anemia and thrombocytopenia $2.6 \%$ and neutropenia with thrombocytopenia $2.3 \%$ ); pancytopenic patients represented only $1.1 \%$ of the whole population.

High serum ferritin levels were shown in $33 \%$ of the patients. To better analyze the incidence of anemia, we divided the cohort studied into three groups according to the degree of malnutrition (Table 3): (1) 58 patients of the total sample (18.2\%) had a BMI $<14.4 \mathrm{~kg} / \mathrm{m}^{2}$ (Group 1); (2) $128(40.3 \%)$ were in a BMI range 14.5-16.5 (Group 2); and (3) 132 (41.5\%) with $16.6<\mathrm{BMl}<18.5 \mathrm{~kg} / \mathrm{m}^{2}$ (Group 3). No significant $(P=0.124)$ age difference was observed, and duration of amenorrhea increased according to the worsening of malnutrition (Group 1: $18.1 \pm 16.1$ months; Group 2: $15.3 \pm 24.7$ months; Group 3: $12.4 \pm 16.3$ months; $P=0.028$ Groups 1 vs 3 ), lasting longer in patients at Group 1. The percent of patients with PA $<5.0^{\circ}$ was significantly higher in Group 1 (44.1\%) compared with Groups 2 (6.2\%; $P<0.000)$ and 3 (25.3\%; $P<0.001)$. Prevalence of anemia was significant when Group 1 was compared with Groups 2 and 3. No statistical difference was found as far as neutropenia prevalence was considered.

Table 1. Main anthropometric data, duration of amenorrhea in 318 female anorexia nervosa patients

\begin{tabular}{lc}
\hline & Mean \pm s.d. (min-max) \\
\hline Age (years) & $20.4 \pm 5.6(12.0-47.0)$ \\
Height $(\mathrm{cm})$ & $160 \pm 6.0(143-177)$ \\
Weight $(\mathrm{kg})$ & $40.9 \pm 5.3(27.0-56.0)$ \\
BMI (normal range: $\left.20-25 \mathrm{~kg} / \mathrm{m}^{2}\right)$ & $15.9 \pm 1.6(10.5-18.4)$ \\
Phase angle (normal range: $\left.5.5^{\circ}-6.5^{\circ}\right)$ & $5.5 \pm 1.0(2.3-8.3)$ \\
Duration of amenorrhea (months) & $14.5 \pm 20.1(3-180)$ \\
\hline Abbreviation: BMl, body mass index. & \\
\hline
\end{tabular}

\section{DISCUSSION}

Malnutrition secondary to eating disorders is, in absence of other complications, a marasmus-like malnutrition where endocrine, metabolic and functional adaptive mechanisms develop allowing survival, and even apparently normal life habits, despite very low body weight and organ/apparatus complications. ${ }^{15}$ Hematological changes in eating disorders have been described previously; ${ }^{16}$ however, their prevalence is still controversial. Discordant data in literature are possibly due to the heterogeneity of patients studied, degree of malnutrition, medical complications, age, ethnicity and so on. ${ }^{1,15,16}$ For these reasons and for early detection of complications in these patients, a careful clinical nutrition monitoring is suggested.

The most exhaustive review published by Hütter et al. ${ }^{1}$ underlines that hematological disorder prevalence can be present in almost one-third of the entire number of patients: anemia and neutropenia being more common, whereas thrombocytopenia is almost rare. Hütter's review compares several studies, from 1988 to 2005 , but only the one by Miller et al. ${ }^{15}$ in 2005 collected a large number (214) of patients recruited by advertisements, but without evaluating other possible causes of anemia and neutropenia. The studies of Hütter and Miller reported a higher prevalence of anemia and neutropenia in a population of young American and northern European patients with AN compared with our results. This could be related to environmental factors and food choice., ${ }^{1,15}$

In the present study, we have analyzed a cohort of 318 noncritical patients with AN. Iron status confirmed that no patient was defective in iron storage, whereas 33\% showed high serum ferritin levels, apparently not secondary to an inflammatory condition. Our sample was homogeneous also concerning its ethnicity: all Caucasians and born in the Campania region, Southern Italy. The selection of our patients, which excluded all other possible causes of anemia, may represent one of the factors that justify the lower incidence of hematological defects observed in comparison with the ones described in another paper. ${ }^{1}$

We cannot exclude that traditional Mediterranean dietary habits may have a protective role in reducing the incidence of hematological alterations, although caloric intake in these patients was really low. We, however, observed that hematological abnormalities in this selected sample of patients with AN were strictly related to the duration of illness, as suggested by the duration of amenorrhea, and the entity of malnutrition defined either by BMI and PA. PA could also be considered as a sensible marker of fat-free mass nutritional status in patients with AN because it identifies extra/intracellular water distribution according to the previous observation. ${ }^{17}$ Furthermore, recent studies, also conducted by our group, identified PA as a prognostic predictor of survival in cancer malnutrition. ${ }^{18-20}$ Interestingly, other studies on hematological disorders in AN in smaller samples of patients from Northern Europe countries ${ }^{21-24}$ and United States ${ }^{25}$ showed an increased prevalence of anemia, leukopenia and thrombocytopenia. In particular, Lambert et al., ${ }^{24}$ despite the small sample size (10 patients with AN), showed a correlation between total body fat mass and bone marrow depletion (serous bone marrow atrophy) evaluated by magnetic resonance imaging and peripheral blood parameters (lower erythrocyte, leukocyte, neutrophil and platelet counts). Contrary to this findings, Misra et al. ${ }^{25}$ were unable to find a correlation between $\mathrm{BMI}$ in patients with $\mathrm{AN}$ and hematological disorders. Finally, Devuyst et al. ${ }^{23}$ found that low BMI in patients with AN or more severe leukopenia had a higher risk of developing severe infectious diseases.

This study concentrated only on anemia as a possible complication of AN. However, other hematological defects reported in our study, other than micronutrient deficiency or increased degradation of blood cells, were possibly due to a perturbation in the bone marrow hematopoietic function, as suggested by the other study. ${ }^{1}$ Those studies found different patterns of bone marrow ranging from hypoplasia to gelatinous transformation. ${ }^{2,26}$ These conditions are usually completely reverted after an adequate nutritional 
Table 2. Hematological parameters in 318 female anorexia nervosa patients

Under minimum (prevalence)

Over maximum (prevalence)

$\mathrm{Hb}$ (n.v. $12-16 \mathrm{~g} / \mathrm{dl}$ )

Red cells (n.v. $4-5.4 \times 10^{6} \mu$ l)

Hematocrit (n.v. 35-48\%)

MCV (n.v. 80-97 fl)

White cells (n.v. $\left.4.5-10.8 \times 10^{3} \mu \mathrm{l}\right)$

Lymphocytes (n.v. 1-4.8 $\times 10^{3} \mu \mathrm{l}$ )

Neutrophils (n.v. $\left.1.5-7 \times 10^{3} \mu \mathrm{l}\right)$

Platelets (n.v. $150-400 \times 10^{3} \mu$ l)

Serum iron (n.v. $45-150 \mu \mathrm{g} / \mathrm{dl}$ )

Ferritin (n.v. 10-120 ng/dl)

Transferrin (n.v. 200-320 mg/dl)

$13.0 \pm 1.2(7.6-16.2)$
$4.2 \pm 0.4(2.5-5.3)$
$38.4 \pm 3.3(23.3-49.0)$
$91.9 \pm 4.4(82.3-108.8)$
$5.1 \pm 1.7(1.8-13.5)$
$2.0 \pm 0.8(0.2-9.8)$
$2.8 \pm 1.4(0.3-11.4)$
$226.2 \pm 63.3(11.0-504.0)$
$84.5 \pm 28.2(18.0-215.0)$
$105.9 \pm 82.9(5.0-600.0)$
$2.3 \pm 0.4(1.0-3.8)$

$13.0 \pm 1.2(7.6-16.2)$

$4.2 \pm 0.4(2.5-5.3)$

$5.1 \pm 1.7(1.8-13.5)$

$2.0 \pm 0.8(0.2-9.8)$

$26.2 \pm 63.3(11.0-504.0)$

$84.5 \pm 28.2(18.0-215.0)$
$105.9 \pm 82.9(5.0-600.0)$

$2.3 \pm 0.4(1.0-3.8)$

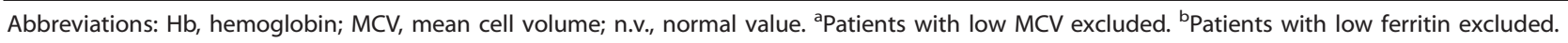

\begin{tabular}{|c|c|c|c|}
\hline Anemia $(\mathrm{Hb}<12 \mathrm{~g} / \mathrm{dl})$ & 19/58 (32.7\%) & $14 / 128^{\mathrm{a}}(10.9 \%)$ & $20 / 132^{\mathrm{b}}(15.2 \%)$ \\
\hline Thrombocytopenia (Plt $\left.<150 \times 10^{3} / \mu \mathrm{l}\right)$ & $5 / 58(8.6 \%)$ & $13 / 128(10.2 \%)$ & $10 / 132(7.6 \%)$ \\
\hline Neutropenia (neutrophils $<1.5 \times 10^{3} / \mu \mathrm{l}$ ) & $8 / 49(16.3 \%)$ & $9 / 106(8.5 \%)$ & $4 / 107^{\mathrm{b}}(3.7 \%)$ \\
\hline
\end{tabular}

Abbreviations: $\mathrm{BMI}$, body mass index; $\mathrm{Hb}$, hemoglobin; PA, phase angle; Plt, platelet. ${ }^{\mathrm{a}} \mathrm{Groups} 2$ vs Group 1: $P<0.001$. ${ }^{\mathrm{b}} \mathrm{Groups} 3$ vs Group 1: $P<0.001$.

rehabilitation with a satisfactory weight regain. ${ }^{1}$ After the exclusion of other causes of cytopenia, it was equally conceivable to hypothesize that a microenvironment perturbation elicited a bone marrow dysplastic alteration. ${ }^{26}$ This alteration could be characterized by a subsequent disequilibrium among the agents cooperating to the hematopoietic niche physiology, where hematopoietic stem cells are driven to differentiation by several interactions with nonhematopoietic cells. Every alteration in this fragile microenvironment could interfere with stem cell differentiation. ${ }^{2,26,27}$

Recently, Zhang and Wang ${ }^{28}$ reported an increase in the preadipocyte factor Pref-1, a member of the epidermal growth factor-like family of proteins and regulator of adipocyte and osteoblast differentiation in patients with $\mathrm{AN}^{28}$ The consequence of this altered Pref-1 production was a defective generation of osteoblasts in the bone marrow. As osteoblasts are strictly related with hematopoiesis, ${ }^{26}$ it is possible to speculate that a reduction in their generation, following Pref- 1 increased secretion, could interfere with hematopoiesis in AN subjects. Furthermore, leptin was suggested to be involved in the development of the hematopoietic bone marrow through erythropoietic-stimulating agents. ${ }^{27,28}$ The imbalance of leptin/Pref-1 ratio could result in another perturbation of the microenvironment with consequent interference with hematopoiesis in patients with AN. However, this hypothesis will require further evaluation.

This study has at least three limitations. The exclusion of patients with low ferritin levels and low MCV helped to avoid the confounding presence of subjects with thalassemia trait. However, it might contribute to under-recording anemic patients with AN Although the clinical records were analyzed carefully, we cannot exclude that some patients had received medication, which could have influenced iron status. Finally, our sample deliberately excluded male subjects, and consequently results of the study may be referred only to female subjects.

In conclusion, our study gives useful information about the incidence of hematologic defects in a large sample of female
Caucasian patients with AN living in southern Italy since 1991 to 2012. In the absence of other complications and clinical emergencies, hematologic changes may be linked to a defective hematopoiesis, possibly due to bone marrow microenvironment alterations. Those alterations might be related to the degree and duration of malnutrition.

These observations require further investigation with intervention studies, particularly for the evaluation of possible environmental and/or nutritional factors that may have affected the relatively lower incidence of hematological impairment observed in our group of patients as opposed to other northern European or American patients with AN.

\section{CONFLICT OF INTEREST}

The authors declare no conflict of interest.

\section{AUTHOR CONTRIBUTIONS}

CDC, FC and MM: designed the research; EDF and FA: conduced this research and wrote paper; PM, MLDG and GC: data collection; FP and MM: statistical analysis and data discussion.

\section{REFERENCES}

1 Hütter G, Ganepola S, Hofmann WK. The hematology of anorexia nervosa. Int J Eat Disord 2009; 42: 293-300.

2 Ozkale M, Sipahi T. Hematologic and bone marrow changes in children with protein-energy malnutrition. Pediatr Hematol Oncol 2014; 31: 349-358.

3 Muhajir M. Gelatinous transformation of bone marrow in a patient with severe anorexia nervosa. Int J Hematol 2013; 97: 157-158.

4 Setnick J. Micronutrient deficiencies and supplementation in anorexia and bulimia nervosa: a review of literature. Nutr Clin Pract 2010; 25: 137-142.

5 Kyle UG, Bosaeus I, De Lorenzo AD, Deurenberg P, Elia M, Manuel Gamez J et al. Bioelectrical impedance analysis-part II: utilization in clinical practice. Clin Nutr 2004; 23: 1430-1453. 
6 Haas V, Riedl A, Hofmann T, Nischan A, Burghardt R, Boschmann M et al. Bioimpedance and bioimpedance vector analysis in patients with anorexia nervosa. Eur Eat Disord Rev 2012; 20: 400-405.

7 Llames L, Baldomero V, Iglesias ML, Rodota LP. Values of the phase angle by bioelectrical impedance; nutritional status and prognostic value. Nutr Hosp 2013; 28: 286-295.

8 Peres WA, Lento DF, Baluz K, Ramalho A. Phase angle as a nutritional evaluation tool in all stages of chronic liver disease. Nutr Hosp 2012; 26: 2072-2078.

9 Kyle UG, Genton L, Pichard C. Low phase angle determined by bioelectrical impedance analysis is associated with malnutrition and nutritional risk at hospital admission. Clin Nutr 2013; 32: 294-299.

10 Castillo MartÃnez L, ColÃn RamÃrez E, Orea Tejeda A, Asensio Lafuente E, Bernal Rosales LP, Rebollar GonzÃlez V et al. Bioelectrical impedance and strength measurements in patients with heart failure: comparison with functional class. Nutrition 2007; 23: 412-418.

11 Santarpia L, Marra M, Montagnese C, Alfonsi L, Pasanisi F, Contaldo F. Prognostic significance of bioelectrical impedance phase angle in advanced cancer: preliminary observations. Nutrition 2009; 25: 930-931.

12 Gupta D, Lammersfeld CA, Vashi PG, King J, Dahlk SL, Grutsch JF et al. Bioelectrical impedance phase angle as a prognostic indicator in breast cancer. BMC Cancer 2008; 8: 249.

13 American Psychiatric Association. Diagnostic and Statistical Manual of Mental Disorders, 4th edn. American Psychiatric Association: Arlington, VA, USA, 2000.

14 American Psychiatric Association. Diagnostic and Statistical Manual of Mental Disorders, 5th edn. American Psychiatric Association: Arlington, VA, USA, 2013

15 Miller KK, Grinspoon SK, Ciampa J, Hier J, Herzog D, Klibanski A. Medical findings in outpatients with anorexia nervosa. Arch Intern Med 2005; 165: 561-566.

16 Mant MJ, Faragher BS. The haematology of anorexia nervosa. Br J Haematol 1972; 23: 737-749.

17 Marra M, Caldara A, Montagnese C, De Filippo E, Pasanisi F, Contaldo F et al. Bioelectrical impedance phase angle in constitutionally lean females, ballet dancers and patients with anorexia nervosa. Eur J Clin Nutr 2009; 63: 905-908.

18 Pavia SI, Borges LR, Halpern-Silveira D, Assunção MCF, Barros AJD, Gonzalez MC. Standardized phase angle from bioelectrical impedance analysis as prognostic factor for survival in patients with cancer. Support Care Cancer 2011; 19: 187-192.

19 Gupta D, Lis CG, Dahlk SL, King J, Vashi PG, Grutsch JF et al. The relationship between bioelectrical impedance phase angle and subjective global assessment in advanced colorectal cancer. Nutr J 2008; 30: 7-19.
20 Gupta D, Lammersfeld CA, Vashi PG, King J, Dahlk SL, Grutsch JF et al. Bioelectrical impedance phase angle in clinical practice: implications for prognosis in stage IIIB and IV non-small cell lung cancer. BMC Cancer 2009; 9: 37.

21 Charania RS, Kern WF, Ckarkrabarty S, Holter J. Successful management of gelatinous transformation of the bone marrow in anorexia nervosa with hematopoietic growth factors. Int J Eat Disord 2011; 44: 469-472.

22 Fazeli PK, Bredella MA, Freedman L, Thomas BJ, Breggia A, Meenaghan E et al. Marrow fat and preadipocyte factor-1 levels decrease with recovery in women with anorexia nervosa. J Bone Miner Res 2012; 27: 1864-1871.

23 Devuyst O, Lambert M, Rodhain J, Lefebvre C, Coche E. Haematological changes and infectious complications in anorexia nervosa: a case-control study. $Q \mathrm{~J}$ Med 1993; 86: 791-799.

24 Lambert M, Hubert C, Depresseux G, Vande Berg B, Thissen JP, Nagant de Deuxchaisnes $C$ et al. Hematological changes in anorexia nervosa are correlated with total body fat mass depletion. Int J Eat Disord 1997; 21: 329-334.

25 Misra M, Aggarwal A, Miller KK, Almazan C, Worley M, Soyka LA et al. Effects of anorexia nervosa on clinical, hematologic, biochemical, and bone density parameters in community-dwelling adolescent girls. Pediatric 2004; 114: 1574-1583.

26 Morrison SJ, Scadden DT. The bone marrow niche for haematopoietic stem cells. Nature 2014; 505: 327-334.

27 Gainsford T, Willson TA, Metcalf D, Handman E, McFarlane C, Ng A et al. Leptin can induce proliferation, differentiation, and functional activation of hemopoietic cells. Proc Natl Acad Sci USA 1996; 93: 14564-14568.

28 Zhang J, Wang N. Leptin in chronic kidney disease: a link between hematopoiesis, bone metabolism, and nutrition. Int Urol Nephrol 2013; 46: 1169-1174.

This work is licensed under a Creative Commons AttributionNonCommercial-NoDerivs 4.0 International License. The images or other third party material in this article are included in the article's Creative Commons license, unless indicated otherwise in the credit line; if the material is not included under the Creative Commons license, users will need to obtain permission from the license holder to reproduce the material. To view a copy of this license, visit http:// creativecommons.org/licenses/by-nc-nd/4.0/

(c) The Author(s) 2016 